\title{
Welches Fachgebiet akademisieren wir derzeit eigentlich an den Universitäten? - Ein Wort zum Terminologie-Wirrwarr!
}

\author{
Dieter Melchart \\ Zentrum für naturheilkundliche Forschung der II. Med. Klinik und Poliklinik, TU München, Deutschland
}

Das Jahr 2008 war ein besonders erfolgreiches Jahr für die wissenschaftliche Verankerung und Akademisierung unseres Fachgebietes an den deutschen medizinischen Fakultäten (vgl. auch [1-3]). Viele Professuren wurden ausgeschrieben, neu geschaffen oder besetzt. Bei näherer Betrachtung dieser Professuren finden sich ohne Ausnahme Hinweise auf den Kontext zur Stiftungsfinanzierung und die Tatsache, dass ein gemeinsamer, einheitlicher Gebietsanspruch fehlt. Welches Fachgebiet wird aber dann derzeit in Forschung, Lehre und Ausübung innerhalb der betroffenen Universitäten akademisiert? Die Terminologie reicht von Naturheilkunde über Komplementärmedizin bis hin zur Integrativen Medizin.

Die Verankerung der Naturheilkunde/Komplementärmedizin «auf Zeit», im Sinne einer Probe-Eingliederung, wird durch das Stiftungspräfix verdeutlicht. Dies kann nicht überraschen, da selbst die Bundesregierung seit Jahren keine explizite Forschungsförderung mehr zur Verfügung oder in Aussicht stellt. An der unterschiedlichen Bezeichnung der wissenschaftlichen Zuständigkeit unseres Fachgebietes sind wir aber selber schuld - es fehlt einfach die Bereitschaft zur Abstimmung und zum gegenseitigen Dialog. Diese uneinheitliche Selbstdarstellung erschwert die Akzeptanz und Integration unseres Fachgebiets in die Medizinischen Fakultäten.

Die Zeitschrift ForschendE KoMPLEMENTÄRMEDIZIN hat es in 15 Jahren Aufbauarbeit durch einen mühsamen und organisierten Dialog geschafft, das Gebiet der klassischen Naturheilkunde und der Komplementärmedizin in einem international anerkannten Publikationsforum mit vorwiegend wissenschaftlichen Nachwuchsvertretern schulmedizinischer Fakultäten zu vereinigen. Es bestehen seit Jahren weder «disziplinäre Eitelkeiten» [4], noch bedarf es der Bezeichnung «Integrative Medizin», um einen Mangel an «Brücke[nschlag] zwischen Schulmedizin und komplementärmedizinischen Verfahren» [5] zu überwinden.

Auch wenn die verstärkte Akademisierung hoffen lässt, dass

\section{Integrative Medicine}

- Combines the best of both conventional and EBM-based CAM therapies

- Emphasizes patient participation (e.g. exercise, diet, stress management) in maximizing health

- Emphasizes the primacy of the patient provider relationship and the importance of shared decision making

- Emphasizes the contribution of the therapeutic encounter itself

- Seeks to optimize an individual's innate healing capacity

Abb. 1. Integrative Medizin (nach Eisenberg aus [6]).

eine terminologische Fortentwicklung folgen wird, ist die bloße Übertragung und aktive Verbreitung der englischen Bezeichnung «Integrative Medicine» - wie es Snyderman und Weil 2002 [6] im renommierten Journal Archives of Internal Medicine sinngemäß definiert haben (vgl. Abb. 1) - in die deutsche Sprachenlandschaft weder aus einer Binnensicht noch aus der Sicht einer Außendarstellung gegenüber der Bevölkerung notwendig und sinnvoll. Die Öffentlichkeit kann sich heute etwas unter einem Naturheilkundigen oder Komplementärmediziner vorstellen - sicher nichts unter dem Begriff eines «Integrativmediziners»!

Die zitierte Beschreibung einer «Integrative Medicine» ist inhaltlich durchaus akzeptabel und nachvollziehbar. Es entzieht sich aber meinem Verständnis, dass ein mühsamer Terminologieprozess von Naturheilkunde und Komplementärmedizin der immerhin dazu geführt hat, dass diese Begriffe im Wörterbuch Naturheilkunde von Pschyrembel [7] definiert werden durch Popularisierung des Begriffs «Integrative Medizin» ohne Not erneut in Wirrnis gebracht wird.

Sozioepidemiologisch betrachtet bedeutet «integrieren» (d.h. «sich zusammenschließen» [8]), ein Signal an die «offizielle Medizin»: Wir wollen mit euch ein «größeres Ganzes» bilden

\section{KARGER}

Fax +497614520714

Information@Karger.de

www.karger.com
() 2008 S. Karger GmbH, Freiburg

Accessible online at:

www.karger.com/fok 
[8]. Dem will keiner widersprechen. Aber es geht nicht nur darum, integriert und eingebunden $\mathrm{zu}$ werden, sondern darum, mit eigenständigen Theorien und Ausführungsstrategien die Medizin zu ergänzen. Die Komplementärmedizin strebt ein Sowohl-als-auch an - angelehnt an den Begriff der Komplementarität nach Niels Bohr. Die Akademisierung der Naturheilkunde und Komplementärmedizin hat die Aufgabe, eigenständige Theorien und Hypothesen mit wissenschaftlich adäquaten Methoden zu überprüfen. Hier sind wichtige Bereiche der Salutogenese, der Placebo- bzw. Kontextfaktorenforschung, der Matrixforschung, der Anthropologie sowie der
Forschungskoordination mit dem Ziel einer transdisziplinären Wissenschaftsstruktur zu bearbeiten. Dieser Ergänzungsaspekt bezieht sich sowohl auf die einzelnen Verfahren als auch auf die fehlende plurale medizinische Versorgung und die unterschiedlichen wissenschaftlichen Sichtweisen.

Diese «Ergänzungsarbeit» sollte unser gemeinsames Ziel und die Basis eines gemeinsamen Selbstverständnisses unseres Fachgebietes darstellen. Zugegeben, es existiert derzeit keine zufriedenstellende Nomenklatur. Es wäre aber die Aufgabe der akademischen Etablierer, diese bald im Konsens zu finden!

\section{Literatur}

1 Joos S, Eicher C, Musselmann B, Kadmon M: Entwicklung, Implementierung und Evaluation eines «Curriculums Naturheilverfahren» an der Universität Heidelberg. Forsch Komplementmed 2008;15: 251-160.

2 Witt CM, Brinkhaus B, Willich SN: Teaching complementary and alternative medicine ina reform curriculum. Forsch Komplementmed 2006;13: 342-348.
3 Brinkhaus B, Joos S, Lindner M, Kohnen R, Witt CM, Willich SN, Hahn EG: Integration of complementary and alternative medicine into Geman medical school curricula - contradictions between the options of decision makers and the status quo. Forsch Komplementärmed Klass Naturheilkd 2005; 12:139-143.

4 Matthiessen Peter F. Pluralität - auf dem Weg zu einer Integrativen Medizin? Forsch Komplementärmedizin 2008;15:2-4.
5 Europäischer Kongress für Integrative Medizin: Einladungsprospekt. www.ecim-congress.org.

6 Snyderman R, Weil AT, Integrative Medicine: Bringing medicine back to the roots. Arch Int Med; 2002;162:395-397

7 Pschyrembel Wörterbuch Naturheilkunde und alternative Heilverfahren, ed 3. Berlin, Gruyter, 2006

8 Deutsches Wörterbuch (Bertelsmann-Lexikon). Gütersloh, Bertelsmann, 2000. 